\title{
DIFFERENTIATION OF SET FUNCTIONS USING VITALI COVERINGS $\left({ }^{1}\right)$
}

BY

\author{
W. E. HARTNETT AND A. H. KRUSE
}

1. Introduction. The original motivation for this paper was a proposal by G. B. Price in which he suggested a way for basing a theory of surface area and more general theories on a measure-theoretic foundation. Previous writers, especially T. Radó [8, p. 552], had already commented on the lack of a measure-theoretic approach to surface area comparable to the Lebesgue theory and had indicated the desirability of such an approach.

Certain results of W. K. Moore [4] concerning generalized derivatives seemed to indicate that under reasonable conditions one could expect to be able to compute the area of a surface as the integral of the derivative of a set function connected with the surface, the integral to be taken with respect to a suitably defined measure.

In this paper only the abstract measure-theoretic tools used in the approach are developed; applications to surface area will be reserved for a later paper, where it will be shown that for an important class of surfaces (see Radó [8, p. 439]), our treatment gives the same result as the classical Lebesgue approach.

We now summarize the paper.

Throughout, $X$ will be a metric space, $B$ will be the set of all Borel sets of $X$, and $\mu$ will be a measure on $B$. Also, $\mathfrak{e}$ will be a set of subsets of $X$, usually a $\mu$-Vitali covering of $X$ (cf. Definition 1 in $\S 2$ ), and $\lambda$ will be an extended-real-valued function whose domain includes $\mathrm{e}$. In $\S 3$ we define the lower measure $m_{\circ}$ (whose domain includes $B$ ) by applying Munroe's Method II (cf. [7, p. 105]) to $\lambda$ and $\mathcal{C}$. In $\S 4$ we define the upper measure $m^{\circ}$ similarly by using open coverings directed by refinement (rather than the explicit metric on $X$ ). In $\$ 5$ we use standard methods to prove that, under certain conditions, the $\mu$-nonsingular component of $m_{\circ}$ is the indefinite integral of the lower derivative of $\lambda$ with respect to $\mu$ and $\mathcal{e}$ (cf. Definition 2 of $\$ 2$ and Theorem 9 and Remark 10 of $\$ 5$ ). In $\S 6$ we use standard methods to prove

Presented to the Society, January 29, 1960; received by the editors April 27, 1959 and, in revised form, October 27, 1959.

(1) Portions of this paper appeared in the doctoral thesis of Hartnett, completed at the University of Kansas under the direction of G. B. Price. Sincere thanks are due to G. B. Price for his guidance and to A. H. Kruse for his encouragement and assistance during the preparation of the thesis. Special thanks go to the National Science Foundation for the financial support given to Hartnett while he was a Research Assistant on NSF Project G 1976 at the University of Kansas from June 1956 to July 1957. The research of Kruse in this paper has been sponsored by the National Science Foundation in connection with the same project. 
that, under certain conditions, $m^{\circ}$ is the integral of the upper derivative of $\lambda$ with respect to $\mu$ and $\mathcal{e}$ (cf. Definition 2 of $\$ 2$ and Theorem 12 of $\S 6$ ). In $\S 7$ we investigate the relationship between $m_{\circ}$ and $m^{\circ}$ and formulate conditions in terms of $m_{\circ}$ and $m^{\circ}$ sufficient for the differentiability of $\lambda$ with respect to $\mu$ and $\mathfrak{e}$ almost everywhere $(\bmod \mu$ ) (cf. Theorem 15 of $\S 7$ and its corollary). We specialize our results to the case in which $\lambda$ is a measure in Theorem 16. In $\S 8$ we specialize to the case in which $X$ is a subset of Euclidean $q$-space and $\mu$ is the appropriate restriction of Lebesgue measure.

2. Preliminaries. We let $X$ be a fixed metric space with metric $d$, and we let $P(X)$ be the class of all subsets of $X$. $B$ is the class of all Borel sets in $X$ and $\mu$ is a measure on $B$ with a regular completion

$$
\tilde{\mu}: B^{*} \rightarrow\{t \mid 0 \leqq t \leqq+\infty\}
$$

where $B^{*}$ is the domain of the completion of $\mu$.

A Baire set (or Borel set) of $X$ is any member of the sigma-ring generated by the set of open sets of $X$. A Baire function is an extended-real-valued function $f$ on $X$ such that $f^{-1}(U)$ is a Baire set for each open set $U$ of real numbers.

Definition 1. A $\mu$-Vitali covering $\left({ }^{2}\right)$ of $X$ is a set $\mathcal{C} \subset \mathbb{B}^{*}$ such that

(1) $\varnothing \in \mathfrak{e}$.

(2) $0<\tilde{\mu}(C)<+\infty$ for each nonvoid $C \in \mathbb{e}$.

(3) For each positive integer $n$ the union of some countable subset of

$$
\mathfrak{e}_{n}=\left\{C \mid C \in \mathfrak{e}, \operatorname{diam}(C) \leqq \frac{1}{n}\right\}
$$

is $X$.

(4) If $D \subset C, D_{n}=\mathfrak{C}_{n} \cap D$ for $n=1,2, \cdots$, and $A \subset \cap_{n=1}^{\infty} \cup_{D \in D_{n}} D$, then there is a sequence $\left\{D_{n}\right\}_{n=1}^{\infty}$ in $D \cup\{\varnothing\}$ such that $D_{m} \cap D_{n}=\varnothing$ for $m \neq n$ and such that $\mu\left(A-\cup_{n=1}^{\infty} D_{n}\right)=0$.

On the line, the set of all closed intervals is an $L$-Vitali covering, where $L$ is the Lebesgue measure in $R^{1}$.

REMARK 1. The existence of a $\mu$-Vitali covering implies that $X$ is separable and that $\mu$ is sigma-finite $\left.{ }^{3}\right)$. To prove $\mu$ is sigma-finite, one could first show from (3) that $\tilde{\mu}$ is sigma-finite. It would then follow that $\mu$ is sigma-finite, for, given $B^{*} \in Q^{*}$, there is a $B \in \beta$ such that $B^{*} C B$ and $\tilde{\mu}\left(B^{*}\right)=\mu(B)$.

Remark 2. Suppose that $X$ is a Borel subset of $R^{q}$, suppose $d$ is the Euclidean metric, and suppose $\mu$ is the restriction of Lebesgue measure to the set of Borel sets of $X$. Suppose $\mathcal{e}$ is a set of compact subsets of $X$ such that (1) and (3) hold and such that for some real number $c>0$,

(2) For a general discussion of $\mu$-Vitali coverings together with more general coverings, in which condition (2) does not appear and (1) is negated, see Hahn-Rosenthal [2, Chapter V, $\S 17]$. Also, in the same regard, see Morse $[5 ; 6]$.

(3) For the definition of sigma-finite see Halmos [3, p. 31]. 


$$
\mu(C)>c[\operatorname{diam}(C)]^{q} \quad \text { for each } C \in \mathbb{e} .
$$

Then $\mathcal{C}$ is a $\mu$-Vitali covering. For the statement of a more general theorem for $R^{q}$ and pertinent bibliography, see Hahn-Rosenthal [2, $\$ 17$, Part 5]. It follows, e.g., that the set of all closed cubes is a $\mu$-Vitali covering if $X=R^{q}$. For the existence of Vitali coverings in a separable metric space, cf. Morse $[5 ; 6]$.

Informally, what we require of the class $\mathfrak{C}$ is that it be such that each subclass which, at each point $t$, contains sets with arbitrarily small diameter which in turn contain $t$, satisfies the conclusion of the Vitali Covering Theorem with respect to $\mu$.

Now let $\mathcal{e}$ be a $\mu$-Vitali covering in $X$, and let $\lambda$ be a non-negative, extended-real-valued-function whose domain includes $\mathfrak{e}$ and which is such that $\lambda(\varnothing)=0$.

Definition 2. The upper e-derivative $D^{\circ}(, \lambda)$ of $\lambda$ at a point $t \in X$ (with respect to $\tilde{\mu}$ ) is defined by

$$
\text { - } D^{\circ}(t, \lambda)=D^{\circ}(t, \lambda ; \mathfrak{C}, \tilde{\mu})=\lim _{t \in C \in \mathcal{C} ; \operatorname{diam}(C) \rightarrow 0} \frac{\lambda(C)}{\tilde{\mu}(C)} .
$$

The lower e-derivative $D_{0}(, \lambda)$ of $\lambda$ at a point $t \in X$ (with respect to $\tilde{\mu}$ ) is defined by

$$
D_{\circ}(t, \lambda)=D_{\circ}(t, \lambda ; \mathfrak{e}, \tilde{\mu})=\liminf _{t \in C \in \mathcal{C} ; \operatorname{diam}(C) \rightarrow 0} \frac{\lambda(C)}{\tilde{\mu}(C)} .
$$

If for some $t \in X, D^{\circ}(t, \lambda)=D_{\circ}(t, \lambda)$, we say that $\lambda$ is -differentiable at $t$ (with respect to $\tilde{\mu}$ ) and we denote the derivative by $D(t, \lambda)$.

An extended-real-valued function $f$ on $X$ is $\tilde{\mu}$-measurable if and only if $f^{-1}(U) \in B^{*}$ for each open set $U$ of real numbers.

Theorem $\left.1{ }^{4}\right) . D_{\circ}(, \lambda)$ and $D^{\circ}(, \lambda)$ are $\tilde{\mu}$-measurable. If each member of $\mathcal{e}$ is open, then $D_{\circ}(, \lambda)$ and $D^{\circ}(, \lambda)$ are Baire functions.

Proof. Suppose $a$ is a real number. Let

$$
A=\left\{t \in X \mid D_{\circ}(t, \lambda) \leqq a\right\} .
$$

For $k=1,2, \cdots$, there is a sequence $\left\{C_{j}(k)\right\}_{j=1}^{\infty}$ of members of $\mathfrak{e}_{k}$ such that

$$
\lambda\left(C_{j}(k)\right) / \tilde{\mu}\left(C_{j}(k)\right)<a+(1 / k) \quad(j=1,2, \cdots)
$$

and

$$
\tilde{\mu}\left(A-\bigcup_{j=1}^{\infty} C_{j}(k)\right)=0 .
$$

(4) Essentially the first part of this theorem is found in Hahn-Rosenthal [2, Theorem 17.2.2, p. 247]. A proof will be indicated here for completeness. 
Let $B=\bigcap_{k=1}^{\infty} \cup_{j=1}^{\infty} C_{j}(k)$. Then $\tilde{\mu}(A-B)=0$. If $t \in B$, then $t \in \bigcap_{k=1}^{\infty} C_{j_{k}}(k)$ for some $j_{k}(k=1,2, \cdots)$,

$$
D_{\circ}(t, \lambda) \leqq \lim \inf \lambda\left(C_{j_{k}}(k)\right) / \tilde{\mu}\left(C_{j_{k}}(k)\right) \leqq a,
$$

and $t \in A$. Since $B \in Q^{*}, B \subset A$, and $\tilde{\mu}(A-B)=0$, we have $A \in B^{*}$. Thus $D_{\circ}(, \lambda)$ is $\tilde{\mu}$-measurable, and similarly $D^{\circ}(, \lambda)$ is $\tilde{\mu}$-measurable. If each member of $\mathfrak{e}$ is open, replace $\bigcup_{j=1}^{\infty} C_{j}(k)$ in the preceding argument by the union of all $C \in \mathbb{C}_{k}$ for which $\lambda(C) / \tilde{\mu}(C)<a+(1 / k)$. Then, modified accordingly, $B$ is a Borel set and $B=A$.

Definition 3. $\lambda$ is absolutely continuous with respect to $\tilde{\mu}$ if, given $\epsilon>0$, there is a $\delta>0$ such that if $\left\{C_{1}, \cdots, C_{n}\right\}$ is any finite collection of members of $\mathfrak{e}$ for which $\tilde{\mu}\left(C_{m} \cap C_{k}\right)=0$ for $k \neq m$, then $\sum_{i=1}^{n} \tilde{\mu}\left(C_{i}\right)<\delta$ implies that $\sum_{i=1}^{n} \lambda\left(C_{i}\right)<\epsilon$.

Definition 4. $\lambda$ is $\tilde{\mu}$-Lipschitzian (on $\mathfrak{C}$ ) if there is a non-negative constant $K$ such that $\lambda(C) \leqq K \tilde{\mu}(C)$ for each $C \in \mathcal{e}$.

REMARK 3. If $\lambda$ is $\tilde{\mu}$-Lipschitzian, then $\lambda$ is absolutely continuous with respect to $\tilde{\mu}$.

Definition 5. The strong upper e-derivative $D^{\circ *}(, \lambda)$ of $\lambda$ at a point $t \in X$ (with respect to $\tilde{\mu}$ ) is defined by

$$
D^{\circ *}(t, \lambda)=D^{\circ *}(t, \lambda ; \mathfrak{C}, \tilde{\mu})=\lim _{\phi \neq C \in \mathcal{C} ; \operatorname{diam}(C \cup[t]) \rightarrow 0} \frac{\lambda(C)}{\tilde{\mu}(C)} .
$$

The strong lower $\mathcal{C}$-derivative $D_{\circ}^{*}(, \lambda)$ of $\lambda$ at a point $t \in X$ (with respect to $\tilde{\mu}$ ) is defined by

$$
D_{\circ}^{*}(t, \lambda)=D_{\circ}^{*}(t, \lambda ; \mathfrak{C}, \tilde{\mu})=\liminf _{\phi \neq C \in \mathcal{C} ; \operatorname{diam}(C \cup(t)) \rightarrow 0} \frac{\lambda(C)}{\tilde{\mu}(C)} .
$$

If $t \in X$ and $D^{\circ *}(t, \lambda)=D_{0}^{*}(t, \lambda)$, we say that $\lambda$ is strongly $\mathcal{C}$-differentiable at $t$ (with respect to $\tilde{\mu}$ ), and $D^{\circ *}(t, \lambda)=D_{\circ}^{*}(t, \lambda)=D^{*}(t, \lambda)$ is the strong ederivative of $\lambda$ at $t$ (with respect to $\tilde{\mu}$ ).

REMARK 4 . It is easily seen that for each $t \in X$ :

$$
\begin{aligned}
D^{\circ}(t, \lambda) & =\max \left\{D^{\circ}(t, \lambda), \underset{s \rightarrow t}{\lim \sup } D^{\circ}(s, \lambda)\right\} ; \\
D_{\circ}^{*}(t, \lambda) & =\min \left\{D_{\circ}(t, \lambda), \liminf _{s \rightarrow t} D_{\circ}(s, \lambda)\right\} .
\end{aligned}
$$

Hence $D^{\circ *}(, \lambda)$ is the smallest upper semi-continuous extended-real-valued function on $X$ which is $\geqq D^{\circ}(, \lambda)$ and $D_{\circ}^{*}(, \lambda)$ is the largest lower semicontinuous extended-real-valued function on $X$ which is $\leqq D_{\circ}(, \lambda)$. Thus $D^{*}(, \lambda)$ is continuous at each point of its domain. $D^{\circ *}(, \lambda)$ and $D_{\circ}^{*}(, \lambda)$ are Borel functions, and hence the domain of $D^{*}(, \lambda)$ is a Borel set.

3. Construction of the lower measure $m_{0}$. In this section M. E. Munroe's Method II (cf. Munroe [9, p. 105]) for the construction of metric outer measures will be sketched for later use. 
Let there be given some subclass $\mathcal{C}$ of $\mathbb{P}(X)$ such that for each $n$ a countable collection of members of $\mathfrak{e}_{n}$ (defined as in (3) of Definition 1 of $\$ 1$ ) covers $X$. We define $\operatorname{diam}(\varnothing)=0$.

Suppose now that we have a function $\lambda$ whose domain includes $\mathfrak{e}$, whose image is contained in the set of non-negative, extended-real-numbers, and which is such that $\lambda(\varnothing)=0$. For each $A \in \mathcal{P}(X)$, let

$$
m_{n}^{*}(A)=\inf \left\{\sum_{i=1}^{\infty} \lambda\left(C_{i}\right) \mid C_{i} \in \mathfrak{e}_{n} \text { for each } i, A \subset \bigcup_{i=1}^{\infty} C_{i}\right\} .
$$

Since $\mathcal{e}_{n+1} \subset \mathcal{C}_{n}$, we have $m_{n}^{*}(A) \leqq m_{n+1}^{*}(A)$ for each $A \in \mathcal{P}(X)$. Hence, as $n \rightarrow \infty,\left\{m_{n}^{*}(A)\right\}_{n=1}^{\infty}$ approaches a limit, finite or infinite, and we define

$$
m_{\circ}^{*}(A)=\lim _{n \rightarrow \infty} m_{n}^{*}(A)=\sup _{n} m_{n}^{*}(A) \quad(A \in \mathcal{P}(X)) .
$$

We then have

THEOREM 2. The set function $m_{\circ}{ }^{*}$ is a metric outer measure $\left({ }^{5}\right)$. In particular, each Borel set of $X$ is $m_{0}{ }^{*}$-measurable $\left(^{6}\right)$.

Definition 6. The outer measure $m_{\circ}^{*}$ will be called the lower outer measure induced by $\lambda$ and $\mathcal{C}$. The restriction of $m_{\circ}{ }^{*}$ to the class of all $m_{\circ}{ }^{*}$-measurable sets will be denoted by $m_{\circ}$ and is called the lower measure generated by $\lambda$ and $\mathrm{e}$.

REMARK 5. The domain of $m_{\circ}$ contains $\&$ by Theorem 2 .

4. Construction of the upper measure $m^{\circ}$. In this section $\mathcal{C}$ is a subset of $\mathcal{P}(X)$ and such that $\varnothing \in \mathcal{C}$, and $\lambda$ is a non-negative and extended-real-valued function whose domain includes $\mathcal{C}$ and is such that $\lambda(\varnothing)=0$.

Definition 7. For each non-void $u \subset \mathcal{P}(X)$, let $\lambda^{\circ}(\mathcal{u})$ $=\sup \left\{\sum_{j=1}^{\infty} \lambda\left(C_{j}\right) \mid C_{j} \in \mathfrak{e}\right.$ for $j=1,2, \cdots ; \tilde{\mu}\left(C_{j} \cap C_{h}\right)=0$ if $j \neq h$; for $j=1,2, \cdots, C_{j} \subset U$ for some $\left.U \in \mathcal{u}\right\}$.

REMARK 6. $\lambda^{\circ}(\mathcal{u}) \geqq 0$ for each $u \subset \mathcal{C}(X)$.

Definition 8. If $\mathfrak{u}$ and $v$ are nonvoid sets contained in $\mathscr{P}(X)$, then $v$ refines $\mathcal{u}$ if and only if each member of $v$ is a subset of a member of $u$.

REMARK 7. Observe that if $v$ refines $u$, then $\lambda^{\circ}(v) \leqq \lambda^{\circ}(\mathcal{u})$.

Definition 9. For each $A \in \mathcal{P}(X)$ let $\operatorname{cov}(A)$ be the directed system of nonvoid coverings of $A$ by open members of $\mathcal{P}(X)$, with $\operatorname{cov}(A)$ directed by refinement. (Then $\{\varnothing\} \in \operatorname{cov}(\varnothing)$, but $\varnothing \notin \operatorname{cov}(\varnothing)$.)

It follows that $\lambda^{\circ}$ is nonincreasing on $\operatorname{cov}(A)$ and converges to a limit. We then define

$$
m^{\circ *}(A)=\lim _{\mathcal{U} \in \operatorname{cov}(A)} \lambda^{\circ}(\mathcal{U})=\inf _{\mathcal{U} \in \operatorname{cov}(A)} \lambda^{\circ}(\mathcal{U}) \quad(A \in \mathcal{P}(X)) .
$$

(5) For the definition of metric outer measure, cf. Munroe [7, pp. 85, 101].

(6) For the definition of $m_{\circ}{ }^{*}$-measurable and the standard theorem used here, cf. Munroe $[7$, pp. 86,104$]$. 
Lemma 1. If $\mathfrak{u}_{j}$ is a nonvoid subset of $\mathcal{P}(X)$ for $j=1,2, \cdots$, then

$$
\lambda^{\circ}\left(\bigcup_{j=1}^{\infty} u_{j}\right) \leqq \sum_{j=1}^{\infty} \lambda^{\circ}\left(u_{j}\right) .
$$

Proof. Let $a_{0}=U_{j=1}^{\infty} u_{j}$ and let $\left\{C_{j}\right\}_{j=1}^{\infty}$ be one of those systems used in the definition of $\lambda^{\circ}\left(a_{0}\right)$. Then (where $N_{i}$ is a non-negative integer or $\infty$ )

$$
\sum_{j=1}^{\infty} \lambda\left(C_{j}\right) \leqq \sum_{i=1}^{\infty} \sum_{j=1}^{N_{i}} \lambda\left(C_{j, i}\right)
$$

where $\left\{C_{j, i}\right\}_{j=1}^{N_{i}}$ is the set of those members of $\left\{C_{j}\right\}_{j=1}^{\infty}$ such that $C_{j, i} \subset U \in \mathfrak{u}_{j}$. Now clearly

$$
\sum_{j=1}^{N_{i}} \lambda\left(C_{j, i}\right) \leqq \lambda^{\circ}\left(\mathcal{u}_{i}\right)
$$

hence

$$
\sum_{j=1}^{\infty} \lambda\left(C_{j}\right) \leqq \sum_{i=1}^{\infty} \lambda^{\circ}\left(\mathcal{U}_{i}\right)
$$

and so

$$
\lambda^{\circ}\left(\bigcup_{j=1}^{\infty} u_{j}\right)=\lambda^{\circ}\left(a_{0}\right)=\sup \left\{\sum_{j=1}^{\infty} \lambda\left(C_{j}\right)\right\} \leqq \sum_{i=1}^{\infty} \lambda^{\circ}\left(\mathcal{u}_{i}\right) .
$$

THEOREM 3. $m^{\circ *}$ is a metric outer measure on $\mathcal{P}(X)$. The domain of its restriction $m^{\circ}$ to the class of $m^{\circ *}$-measurable sets contains $B$.

Proof. We first show that $m^{\circ *}$ is an outer measure and then that it is a metric outer measure $\left({ }^{7}\right)$. Since

$$
0 \leqq m^{\circ *}(\varnothing) \leqq \lambda^{\circ}(\{\varnothing\})=0, \quad m^{\circ *}(\varnothing)=0 .
$$

Suppose $A \subset \bigcup_{j=1}^{\infty} A_{j} \in \mathcal{P}(X)$, and suppose $\epsilon>0$. For $j=1,2, \cdots$, there is a $\mathfrak{u}_{j} \in \operatorname{cov}\left(A_{j}\right)$ such that $\lambda^{\circ}\left(\mathcal{u}_{j}\right) \leqq m^{\circ *}\left(A_{j}\right)+2^{-j} \cdot \epsilon$. Then $\bigcup_{j=1}^{\infty} \mathfrak{u}_{j} \in \operatorname{cov}(A)$ and

$$
\begin{aligned}
m^{\circ *}(A) & \leqq \lambda^{\circ}\left(\bigcup_{j=1}^{\infty} \mathfrak{u}_{j}\right) \leqq \sum_{j=1}^{\infty} \lambda^{\circ}\left(\mathcal{u}_{j}\right) \\
& \leqq \sum_{j=1}^{\infty}\left[m^{\circ *}\left(A_{j}\right)+2^{-j} \cdot \epsilon\right]=\sum_{j=1}^{\infty} m^{\circ *}\left(A_{j}\right)+\epsilon .
\end{aligned}
$$

Thus since $\epsilon$ is arbitrary

(7) See Theorem 2. 


$$
m^{\circ *}(A) \leqq \sum_{j=1}^{\infty} m^{\circ *}\left(A_{j}\right) .
$$

In particular, $\bigcup_{j=1}^{\infty} A_{j} \subset \bigcup_{j=1}^{\infty} A_{j}$; so $m^{\circ *}\left(\bigcup_{j=1}^{\infty} A_{j}\right) \leqq \sum_{j=1}^{\infty} m^{\circ *}\left(A_{j}\right)$. Hence $m^{\circ *}$ is an outer measure.

Suppose now that $A, B \in \rho(X)$, and $d(A, B)>0$. For some real $\delta>0$, we have that $d(A, B)>2 \delta$. Let $\epsilon>0$ be given. Then there is a $\mathcal{u} \in \operatorname{cov}(A \cup B)$ such that $\operatorname{diam}(U)<\delta$ and $U \cap(A \cup B) \neq \varnothing$ for each nonvoid $U \in \mathcal{U}$ and such that

$$
m^{\circ *}(A \cup B)+\epsilon>\lambda^{\circ}(\mathcal{U})
$$

But if $\operatorname{diam}(U)<\delta$ for each $U \in \mathfrak{u}$, then $\mathcal{u}$ decomposes into two parts $\mathfrak{u}_{A}$ and $\mathcal{u}_{B}$ so that

$$
\mathfrak{u}=\mathfrak{u}_{A} \cup \mathfrak{u}_{B}
$$

where

$$
\mathfrak{u}_{A} \in \operatorname{cov}(A), \quad \mathfrak{u}_{B} \in \operatorname{cov}(B)
$$

and

$$
U \cap V=\varnothing \text { for all } U \in \mathcal{u}_{A} \text { and } V \in \mathfrak{u}_{B} \text {. }
$$

Hence

$$
m^{\circ *}(A \cup B)+\epsilon>\lambda^{\circ}\left(\mathcal{U}_{A}\right)+\lambda^{\circ}\left(\mathcal{U}_{B}\right) \geqq m^{\circ *}(A)+m^{\circ *}(B) .
$$

Since $\epsilon>0$ is arbitrary, we have

$$
m^{\circ *}(A \cup B) \geqq m^{\circ *}(A)+m^{\circ *}(B) .
$$

Hence $m^{\circ *}$ is a metric outer measure.

The proof of the following theorem is routine.

THEOREM 4. Suppose $\mu$ is regular $\left(^{8}\right)$, and suppose that $\lambda \mid \mathfrak{C}$ is absolutely continuous with respect to $\tilde{\mu}$. Then each member of $B^{*}$ is $m^{\circ *}$-measurable, and $m^{\circ} \mid B^{*}$ is absolutely continuous with respect to $\tilde{\mu}$.

In the remainder of this section $\mathcal{C}$ and $\lambda$ will be as stipulated in $\S 2$. The metric outer measure $m_{\circ} *$ of $\$ 3$ will be shown to be the same as an outer measure about to be defined by means of coverings.

Definition 10. For each $A \subset X$ and each nonvoid $\mathfrak{u} \in \operatorname{cov}(A)$, let $\lambda_{\circ}(u ; A)=\inf \left\{\sum_{j=1}^{\infty} \lambda\left(C_{j}\right) \mid C_{j} \in \mathcal{e}\right.$ for $j=1,2, \cdots ; A \subset \bigcup_{j=1}^{\infty} C_{j} ;$ for $j=1,2$, $\cdots, C_{j} \subset U$ for some $\left.U \in \mathcal{u}\right\}$.

Then $\lambda_{\circ}(; A)$ is nondecreasing under refinement. For each $A \subset X$, let

$$
\bar{m}_{\circ}^{*}(A)=\lim _{\mathcal{U} \in \operatorname{cov}(A)} \lambda_{\circ}(\mathcal{U} ; A)=\sup _{\mathcal{U} \in \operatorname{cov}(A)} \lambda_{\circ}(\mathcal{U} ; A) .
$$

(8) Cf. $\$ 5$, footnote $\left({ }^{9}\right)$. 
The proof of the following lemma is straightforward. The details will be omitted.

Lemma 2. Suppose $A_{n} \subset X$ and $\mathfrak{u}_{n} \in \operatorname{cov}\left(A_{n}\right)$ for $n=1,2, \cdots$. Let $A \subset \cup_{n=1}^{\infty} A_{n}$, and let $\mathfrak{u} \in \operatorname{cov}(A)$ be such that $\mathfrak{u}_{n}$ refines $\mathfrak{u}$ for $n=1,2, \cdots$. Then $\lambda_{\circ}(\mathcal{u} ; A) \leqq \sum_{n=1}^{\infty} \lambda_{\circ}\left(\mathcal{u}_{n} ; A_{n}\right)$.

The preceding lemma specializes to the finite case via $A_{n}=\varnothing$ and $u_{n}=\{\varnothing\}$ for all sufficiently large $n$.

If $\mathfrak{u}_{n}$ is the set of all open subsets of $X$ of diameter $\leqq 1 / n$, then, for each $A \subset X$,

$$
m_{n}^{*}(A) \leqq \lambda_{\circ}\left(\mathcal{U}_{n} ; A\right) \leqq m_{n+1}^{*}(A)
$$

for $n=1,2, \cdots$, and

$$
m_{\circ}^{*}(A)=\lim _{n \rightarrow \infty} \lambda_{\circ}\left(\mathcal{U}_{n} ; A\right) \leqq \lim _{\mathcal{U} \in \operatorname{cov}(A)} \lambda_{\circ}(\mathcal{U} ; A)=\bar{m}_{\circ}^{*}(A) .
$$

The following theorem will be proved by showing that $\bar{m}_{\mathrm{o}}{ }^{*}(A) \leqq m_{\mathrm{o}}{ }^{*}(A)$.

ThEOREM 5. $\bar{m}_{\circ}^{*}=m_{\circ} *$.

Proof. Suppose $A \subset X$ and $u \in \operatorname{cov}(A)$. For $n=1,2, \cdots$, let $U_{n}$ $=\{t \in X \mid$ for some $r>1 / n$, the open sphere about $t$ of radius $r$ is a subset of a member of $u\}$.

Then $U_{n}$ is open and $\bar{U}_{n} \subset U_{n+1}$ for $n=1,2, \cdots$, and

$$
A \subset \bigcup_{n=1}^{\infty} U_{n}=\bigcup_{U \in \mathcal{U}} U \text {. }
$$

Let $U_{0}=\varnothing$. For $n=1,2, \cdots$, let $\mho_{n}$ be the set of all open subsets of $X$ of diameter $\leqq 1 / n$ which meet $U_{n}$, and let $\mathfrak{u}_{n}=\vartheta_{n} \cup\{\varnothing\}$. Then $\mathfrak{u}_{n}$ $\in \operatorname{cov}\left(A \cap\left(U_{n}-U_{n-1}\right)\right)$ refines $u$, and

$$
\lambda_{\circ}\left(\mathcal{U}_{n} ; A \cap\left(U_{n}-U_{n-1}\right)\right) \leqq m_{n+1}^{*}\left(A \cap\left(U_{n}-U_{n-1}\right)\right)
$$

for $n=1,2, \cdots$. By Lemma 2 ,

$$
\begin{aligned}
\lambda_{\circ}(\mathcal{U} ; A) & \leqq \sum_{n=1}^{\infty} \lambda_{\circ}\left(\mathcal{u}_{n} ; A \cap\left(U_{n}-U_{n-1}\right)\right) \\
& \leqq \sum_{n=1}^{\infty} m_{n+1}^{*}\left(A \cap\left(U_{n}-U_{n-1}\right)\right) \\
& \leqq \sum_{n=1}^{\infty} m_{\circ}^{*}\left(A \cap\left(U_{n}-U_{n-1}\right)\right) \\
& =m_{\circ}^{*}(A)
\end{aligned}
$$


Hence

$$
\bar{m}_{\circ}^{*}(A)=\sup _{\mathcal{U} \in \operatorname{cov}(A)} \lambda_{\circ}(\mathcal{U} ; A) \leqq m_{\circ}^{*}(A) .
$$

The proof is complete.

REMARK 8. It follows from the preceding theorem that $\bar{m}_{\circ} *$ is a metric outer measure. This can be proved directly without reduction to the corresponding result for $m_{\circ}{ }^{*}$. The direct proof would follow the pattern of the proof of the corresponding result for $m_{\circ} *$.

5. Integral representation of $m_{\circ}$. In this section $\mathfrak{e}$ is a $\mu$-Vitali covering of $X$, and $\lambda$ is a non-negative extended-real-valued function whose domain includes $\mathcal{C}$ and which is such that $\lambda(\varnothing)=0$. Then the conditions placed on $\mathcal{C}$ and $\lambda$ in $\$ 2$ and $\S 3$ hold, and $D^{\circ}(, \lambda)$ and $D_{\circ}(, \lambda)$ are defined as in $\S 2$. It will be assumed also that $\mu$ is regular $\left({ }^{9}\right)$.

Remark 9. Suppose $\nu$ is a measure defined on the set of Borel sets of a separable metric space $S$. Then $\nu$ is regular if and only if each $s \in S$ has a neighborhood $N$ such that $\nu(N-\{s\})$ is finite $\left({ }^{10}\right) . \nu$ is called point-finite if $\nu(\{s\})$ is finite for each $s \in S$ and is called locally finite if each $s \in S$ has a neighborhood $N$ such that $\nu(N)$ is infinite. Thus, if $\nu$ is point-finite, then $\nu$ is regular if and only if $\nu$ is locally finite. Since the existence of a $\mu$-Vitali covering implies that $\mu$ is point-finite and $X$ separable, the assumption that $\mu$ is regular is equivalent (under the previous assumption that $\mathfrak{C}$ is a $\mu$-Vitali covering of $X$ ) with the condition that $\mu$ is locally finite.

Lemma 3. Let $B \in Q^{*}$, and suppose $K$ is a non-negative real number such that $D_{\circ}(t, \lambda)<K$ for all $t \in B$. If $\nu: B \rightarrow\{t \mid 0 \leqq t \leqq \infty\}$ is a measure which is continuous $\left.{ }^{11}\right)$ with respect to $\mu$ and $\nu(A) \leqq m_{\circ}(A)$ for all $A \in B$, then for the completion $\tilde{\nu}$ of $\nu$, we have $\tilde{\nu}(B) \leqq K \tilde{\mu}(B)$. (The domain of the completion $\tilde{\nu}$ contains $\mathbb{B}^{*}$ by continuity.)

Proof. Suppose $\epsilon>0$ is given. Since $\tilde{\mu}$ is regular, there is an open set $U$ such that $B \subset U$ and $\tilde{\mu}(U)<\tilde{\mu}(B)+\epsilon$. For each $t \in B$, there is a sequence $\left\{C_{n}(t)\right\}_{n=1}^{\infty}$ in $\mathcal{C}$ such that

$$
\lim _{n \rightarrow \infty} \operatorname{diam}\left(C_{n}(t)\right)=0,
$$

and such that

$$
t \in C_{n}(t) \subset U, \frac{\lambda\left(C_{n}(t)\right)}{\tilde{\mu}\left(C_{n}(t)\right)}<K \quad \text { for } n=1,2, \cdots
$$

${ }^{(9)} \mu$ is regular if and only if for each set $B$ in the domain of $\mu$ and each real $\epsilon>0$ there is an open set $U \subset X$ such that $B C U$ and $\mu(U-B)<\epsilon$. If $\mu$ is regular, so is $\tilde{\mu}$.

${ }^{(10)}$ To prove this, apply the proofs in Halmos [3, p. 52] within open sets of finite measure, and use second countability in the obvious way.

(ii) We say that $\nu$ is continuous with respect to $\mu$ if for each $A$ in the domain of $\mu$ for which $\mu(A)=0, A$ is in the domain of $\nu$ and $\nu(A)=0$. 
From the definition of $\mathcal{e}$, for $k=1,2, \cdots$, there are sequences $\left\{D_{j}(k)\right\}_{j=1}^{\infty}$ such that each nonvoid $D_{j}(k)$ is one of the sets $C_{n}(t)$, such that $D_{j}(k) \cap D_{h}(k)$ $=\varnothing$ for $j \neq h$, such that $D_{j}(k) \in \mathfrak{C}_{k}$ for all $j$ and $k$, and such that $\mu\left(B-\cup_{j=1}^{\infty} D_{j}(k)\right)$ $=0$. Let $B_{0}=\bigcap_{k=1}^{\infty} \cup_{j=1}^{\infty} D_{j}(k)$. Then $\mu\left(B-B_{0}\right)=0$ and hence $\tilde{\nu}\left(B-B_{0}\right)=0$. We then have that

$$
\begin{aligned}
\tilde{\nu}(B) & \leqq \tilde{\nu}\left(B_{0}\right)+\tilde{\nu}\left(B-B_{0}\right)=\tilde{\nu}\left(B_{0}\right) \leqq m_{\circ}\left(B_{0}\right) \\
& \leqq \liminf _{k \rightarrow \infty} \sum_{j=1}^{\infty} \lambda\left(D_{j}(k)\right) \leqq \liminf _{k \rightarrow \infty} \sum_{j=1}^{\infty} K \tilde{\mu}\left(D_{j}(k)\right) \\
& \leqq \liminf _{k \rightarrow \infty} \tilde{\mu}(U) \leqq K(\tilde{\mu}(B)+\epsilon) .
\end{aligned}
$$

Since $\epsilon>0$ is arbitrary, $\tilde{\nu}(B) \leqq K \tilde{\mu}(B)$.

Lemma 4. Let $B \in B^{*}$. Let $\tilde{\mu}(B)<+\infty$, and let $K$ be a non-negative real number such that $D(t, \lambda)<K$ for all $t \in B$. If $\nu: B \rightarrow\{t \mid 0 \leqq t \leqq \infty\}$ is a measure which is continuous with respect to $\mu$ and $\nu(A) \leqq m_{\circ}(A)$ for all $A \in \Theta$, then ( $\tilde{\nu}$ being the completion of $\nu$ )

$$
\tilde{\nu}(B) \leqq \int_{B} D_{\circ}(, \lambda) d \tilde{\mu} .
$$

Proof. For each positive integer $k$ and $j=1,2, \cdots, k$, let

$$
B(j, k)=\left\{t \mid t \in B,(j-1) K / k \leqq D_{\circ}(t, \lambda)<j K / k\right\} .
$$

For $k=1,2, \cdots$, let

$$
f_{k}=\sum_{j=1}^{k} \frac{j K}{k} C_{B(j, k)}
$$

where $C_{B(j, k)}$ is the characteristic function of $B(j, k)$. Then $f_{k}$ is $\tilde{\mu}$-measurable for each $k$, and $\left\{f_{k}\right\}_{k=1}^{\infty}$ converges uniformly to $D_{\circ}(, \lambda)$ on $B$ as $k \rightarrow \infty$. Hence

$$
\lim _{k \rightarrow \infty} \int_{B} f_{k} d \tilde{\mu}=\int_{B} D_{\circ}(, \lambda) d \tilde{\mu} .
$$

Using the definition of $B(j, k)$ and applying Lemma 3 to each of the $B(j, k)$ we obtain

$$
\begin{aligned}
\tilde{\nu}(B) & =\sum_{j=1}^{k} \tilde{\nu}(B(j, k)) \leqq \sum_{j=1}^{k} \frac{j K}{k} \tilde{\mu}(B(j, k)) & \\
& =\int_{B} f_{k} d \tilde{\mu} & \text { for } k=1,2, \cdots
\end{aligned}
$$

Hence 
1960] DIFFERENTIATION OF SET FUNCTIONS USING VITALI COVERINGS 195

$$
\tilde{\nu}(B) \leqq \lim _{\boldsymbol{k} \rightarrow \infty} \int_{B} f_{k} d \tilde{\mu}=\int_{B} D_{\circ}(, \lambda) d \tilde{\mu} .
$$

THEOREM 6. If $\nu: B \rightarrow\{t \mid 0 \leqq t \leqq \infty\}$ is a measure which is continuous with respect to $\mu$ and $\nu(B) \leqq m_{\circ}(B)$ for each $B \in B$, then ( $\tilde{\nu}$ being the completion of $\nu$ )

$$
\tilde{\nu}(B) \leqq \int_{B} D_{\circ}(, \lambda) d \tilde{\mu} \quad \text { for all } B \in B^{*} .
$$

Proof. Suppose $B \in Q^{*}$. We may presume $\int_{B} D_{0}(, \lambda) d \tilde{\mu}<+\infty$, for otherwise the inequality of Theorem 6 is trivial. For $n=1,2, \cdots$, let

$$
\begin{aligned}
A_{n} & =\left\{t \mid t \in B, \frac{1}{n+1} \leqq D_{\circ}(t, \lambda)<\frac{1}{n}\right\} . \\
B_{0} & =\left\{t \mid t \in B, 0<D_{\circ}(t, \lambda)<+\infty\right\} . \\
B_{n} & =\left\{t \mid t \in B, n \leqq D_{\circ}(t, \lambda)<n+1\right\} . \\
B^{\prime} & =\left\{t \mid t \in B, D_{\circ}(t, \lambda)=0\right\} . \\
B^{\prime \prime} & =\left\{t \mid t \in B, D_{\circ}(t, \lambda)=+\infty\right\} .
\end{aligned}
$$

Then, for $n=1,2, \cdots$,

$$
\begin{aligned}
& \tilde{\mu}\left(A_{n}\right) \leqq(n+1) \int_{A_{n}} D_{\circ}(, \lambda) d \tilde{\mu}<+\infty, \\
& \tilde{\mu}\left(B_{n}\right) \leqq \frac{1}{n} \int_{B_{n}} D_{\circ}(, \lambda) d \tilde{\mu}<+\infty .
\end{aligned}
$$

Since $\mu$ is sigma-finite, Lemma 4 may be applied countably of ten to yield

$$
\tilde{\nu}\left(B^{\prime}\right) \leqq \int_{B^{\prime}} D_{0}(, \lambda) d \tilde{\mu}=0 .
$$

Also $B_{0}$ is the disjoint union of the $A_{n}$ 's and the $B_{n}$ 's with $n>0$. Hence, by Lemma 4 ,

$$
\begin{aligned}
\tilde{\nu}\left(B_{0}\right) & =\sum_{n=1}^{\infty}\left\{\tilde{\nu}\left(A_{n}\right)+\tilde{\nu}\left(B_{n}\right)\right\} \\
& \leqq \sum_{n=1}^{\infty}\left\{\int_{A_{n}} D_{0}(, \lambda) d \tilde{\mu}+\int_{B} D_{0}(, \lambda) d \tilde{\mu}\right\} \\
& =\int_{B_{0}} D_{0}(, \lambda) d \tilde{\mu} .
\end{aligned}
$$

If $\tilde{\mu}\left(B^{\prime \prime}\right)=0$, then 


$$
\tilde{\nu}\left(B_{0}\right)+\tilde{\nu}(B)+\tilde{\nu}\left(B^{\prime \prime}\right)=\tilde{\nu}\left(B_{0}\right) \leqq \int_{B_{0}} D_{\circ}(, \lambda) d \tilde{\mu} \leqq \int_{B} D_{\circ}(, \lambda) d \tilde{\mu} .
$$

If $\tilde{\nu}\left(B^{\prime \prime}\right)>0$, then

$$
\tilde{\nu}(B) \leqq \int_{B} D_{0}(, \lambda) d \tilde{\mu}=+\infty .
$$

TheOREM 7. $\int_{B} D_{\circ}(, \lambda) d \tilde{\mu} \leqq m_{\circ}(B)$ for all $B \in B\left({ }^{12}\right)$.

Proof. Suppose $B \in B$. For $k=1,2, \cdots$, there is a sequence $\left\{C_{n}(k)\right\}_{n=1}^{\infty}$ in $\mathfrak{e}_{k}$ such that $B \subset \bigcup_{n=1}^{\infty} C_{n}(k)$ and such that

$$
\sum_{n=1}^{\infty} \lambda\left(C_{n}(k)\right) \leqq m_{\circ}(B)+2^{-k}
$$

For $k=1,2, \cdots, \operatorname{let}\left({ }^{13}\right)$

$$
f_{k}=\sum_{n=1}^{\infty} \frac{\lambda\left(C_{n}(k)\right)}{\tilde{\mu}\left(C_{n}(k)\right)} C_{C_{n}(k)}
$$

where $C_{C_{n}(k)}$ is the characteristic function of $C_{n}(k)$. Then

$$
D_{\circ}(t, \lambda) \leqq \liminf _{k \rightarrow \infty} f_{k}(t) \quad \text { for each } t \in B
$$

Hence, by Fatou's Lemma( $\left.{ }^{14}\right)$

$$
\begin{aligned}
\int_{B} D_{\circ}(, \lambda) d \tilde{\mu} & \leqq \int_{B} \liminf _{k \rightarrow \infty} f_{k} d \tilde{\mu} \\
& \leqq \liminf _{k \rightarrow \infty} \int_{B} f_{k} d \tilde{\mu}=\liminf _{k \rightarrow \infty} \sum_{n=1}^{\infty} \frac{\lambda\left(C_{n}(k)\right)}{\tilde{\mu}\left(C_{n}(k)\right)} \tilde{\mu}\left(C_{n}(k)\right) \\
& =\liminf _{k \rightarrow \infty} \sum_{n=1}^{\infty} \lambda\left(C_{n}(k)\right) \leqq \liminf _{k \rightarrow \infty}\left(m_{\circ}(B)+2^{-k}\right)=m_{\circ}(B)
\end{aligned}
$$

Theorem 8. Suppose that $D_{\circ}(, \lambda)$ is $\tilde{\mu}$-integrable over $X$. Then there is a maximum measure $\nu$ on $B$ such that $\nu$ is absolutely continuous $\left({ }^{15}\right)$ with respect to $\mu$ and $\nu(B) \leqq m_{\circ}(B)$ for each $B \in B$. It is the measure $m_{\circ 0}$ on $B$ given by

$$
m_{\circ 0}(B)=\int_{B} D_{\circ}(, \lambda) d \tilde{\mu} \quad \text { for all } B \in Q
$$

(12) In this theorem and its proof the assumption that $\mu$ is regular may be dispensed with.

(13) Regard $\lambda\left(C_{n}(k)\right) / \tilde{\mu}\left(C_{n}(k)\right)$ as 0 if $C_{n}(k)=\varnothing$, i.e., if $\tilde{\mu}\left(C_{n}(k)\right)=0$.

(14) See Munroe [7, Corollary 27.1.1, p. 191].

(15) We say that a measure $\nu$ is absolutely continuous with respect to a measure $\mu$ if the domain of $\nu$ is part of the domain of $\mu$ and if $\epsilon>0$ implies the existence of a $\delta>0$ such that if $\nu(A)$ is defined, then $\nu(A)<\epsilon$ whenever $\mu(A)<\delta$. 
Proof. $m_{\circ 0}$ is absolutely continuous with respect to $\mu$, and $m_{\circ 0}(B) \leqq m_{\circ}(B)$ by Theorem 7 . The maximum property of $m_{\circ 0}$ follows from Theorem 6 .

Lemma 5. Suppose $\nu$ is a sigma-finite measure on the sigma-algebra $S$ of certain subsets of $S \in S$. Suppose $f$ is an extended-real-valued $\nu$-measurable function on $\mathrm{S}$. Then $f$ is $\nu$-sigma-integrable $\left({ }^{16}\right)$ if and only if $f$ is finite almost everywhere $(\bmod \nu)$.

Proof. $S=\cup_{n=1}^{\infty} S_{n}$ with $S_{n} \in \mathcal{S}$ and $\nu\left(S_{n}\right)<+\infty$ for $n=1,2, \cdots$. Suppose $f$ is finite almost everywhere $(\bmod \nu)$. Let

$$
A_{0}=\{x \in S \mid f(x)=+\infty\} \text {. }
$$

Then $\nu\left(A_{0}\right)=0$. Let

$$
A_{n}=\{x \in S \mid f(x)<n\}
$$

for $n=1,2, \cdots$. Then

$$
S=\bigcup_{n=1}^{\infty} \bigcup_{k=0}^{\infty}\left(S_{n} \cap A_{k}\right)
$$

and $f$ is $\nu$-integrable over $S_{n} \cap A_{k}$ for $n=1,2, \cdots$, for $k=0,1, \cdots$. Thus $f$ is $\nu$-sigma-integrable. One part of the lemma follows; the other part is trivial.

TheOREM 9. Suppose $D_{0}(, \lambda)$ is finite almost everywhere $(\bmod \tilde{\mu})$. Then there is a maximum measure $\nu$ on $B$ such that $\nu$ is continuous with respect to $\mu$ and $\nu(B) \leqq m_{\circ}(B)$ for each $B \in B$. It is the measure $m_{\circ 0}$ on $B$ given by $m_{\circ 0}(B)$ $=\int_{B} D_{0}(, \lambda) d \tilde{\mu}$ for all $B \in B$.

Proof. It follows from Lemma 5 that $m_{\circ 0}$ is continuous with respect to $\tilde{\mu}$, and $m_{\circ 0} \leqq m_{\circ}$ by Theorem 7 . The maximum property of $m_{\circ 0}$ follows from Theorem 6.

REMARK 10. In Theorems 8 and $9, m_{\circ 0}$ may be called the $\tilde{\mu}$-nonsingular component $\left({ }^{17}\right)$ of $m_{0}$.

THEOREM 10. If $m_{\circ}$ is continuous with respect to $\tilde{\mu}$, then $m_{\circ}(B)=\int_{B} D_{\circ}(, \lambda) d \tilde{\mu}$ for all $B \in B^{*}$.

Proof. Apply Theorems 6 and 7 with $\nu=m_{\circ}$.

6. Integral representation of $m^{\circ}$. In this section $\mathcal{C}$ and $\lambda$ are assumed to be given as at the beginning of $\S 5$, but $\mu$ will not be assumed to be regular.

(16) $f$ is $\nu$-sigma-integrable if and only if there is a sequence $\left\{S_{n}\right\}_{n=1}^{\infty}$ of members of $S$ such that $S=\bigcup_{n=1}^{\infty} S_{n}$ and $\int_{S_{n}}|f| d \nu<+\infty$ for $n=1,2, \cdots$.

(17) If $m_{\circ}$ is sigma-finite, then $m_{\circ 0}$ is the nonsingular component in a Lebesgue decomposition of $m_{\circ}$ with respect to $\mu$, which exists (uniquely) by a standard theorem (cf., e.g., Halmos $[3$, p. 134]). The standard theorem on the existence of Lebesgue decomposition does not give Theorems 8 and 9 since $m_{\circ}$ is not assumed to be sigma-finite. 
Lemma 6. Let $B \in \Theta$, and suppose $K$ is a real number such that $D^{\circ}(t, \lambda)>K$ for all $t \in B$. Then $m^{\circ}(B) \geqq K \mu(B)$.

Proof. Suppose $u \in \operatorname{cov}(B)$. For each $t \in B$, there is a sequence $\left\{C_{n}(t)\right\}_{n=1}^{\infty}$ in $\mathcal{C}$ such that $\lim _{n \rightarrow \infty}\left[\operatorname{diam}\left(C_{n}(t)\right)\right]=0, t \in C_{n}(t) \subset U$ for some $U \in \mathcal{U}$ and

$$
\frac{\lambda\left(C_{n}(t)\right)}{\tilde{\mu}\left(C_{n}(t)\right)}>K \quad \text { for } n=1,2, \cdots .
$$

Because $\mathcal{C}$ is a $\mu$-Vitali covering, there is a sequence $\left\{D_{j}\right\}_{j=1}^{\infty}$ such that each nonvoid $D_{j}$ is one of the sets $C_{n}(t)$, such that $D_{j} \cap D_{h}=\varnothing$ for $j \neq h$, and such that $\tilde{\mu}\left(B-B_{0}\right)=0$ where $B_{0}=\bigcup_{j=1}^{\infty} D_{j}$. Then

$$
\begin{aligned}
\lambda^{\circ}(\mathcal{U}) & \geqq \sum_{j=1}^{\infty} \lambda\left(D_{j}\right)>\sum_{j=1}^{\infty} K \tilde{\mu}\left(D_{j}\right) \\
& \geqq K \tilde{\mu}\left(B_{0}\right)=K \tilde{\mu}\left(B_{0}\right)+K \tilde{\mu}\left(B-B_{0}\right) \\
& =K \tilde{\mu}(B)=K \mu(B) .
\end{aligned}
$$

Finally

$$
m^{\circ}(B)=\lim _{\mathcal{U} \in \operatorname{cov}(B)} \lambda^{\circ}(\mathcal{U}) \geqq K \mu(B) .
$$

Lemma 7. Let $B \in \Theta$, let $\mu(B)<+\infty$, and let $K$ be a real number such that $D^{\circ}(t, \lambda)<K$ for all $t \in B$. Then

$$
m^{\circ}(B) \geqq \int_{B} D^{\circ}(, \lambda) d \tilde{\mu} .
$$

Proof. For each positive integer $k$ and $j=1,2, \cdots, k$, let

$$
B(j, k)=\left\{t \mid t \in B,(j-1) K / k \leqq D^{\circ}(t, \lambda)<j K / k\right\} .
$$

For $k=1,2, \cdots$, let

$$
f_{k}=\sum_{j=1}^{k} \frac{(j-1) K}{k} C_{B(j, k)}
$$

where $C_{B(j, k)}$ is the characteristic function of $B(j, k)$. Then $f_{k}$ is $\tilde{\mu}$-measurable for each $k$ and converges uniformly to $D^{\circ}(, \lambda)$ on $B$ as $k \rightarrow \infty$. Thus

$$
\int_{B} D^{\circ}(, \lambda) d \tilde{\mu}=\lim _{k \rightarrow \infty} \int_{B} f_{k} d \tilde{\mu} .
$$

By Lemma 6 applied to $B(j, k)$, and from the definition of $B(j, k)$,

$$
m^{\circ}(B) \geqq \sum_{j=1}^{k} m^{\circ}(B(j, k)) \geqq \sum_{j=1}^{k} \frac{(j-1) K}{k} \tilde{\mu}(B(j, k))=\int_{B} f_{k} d \tilde{\mu}
$$


for $k=1,2, \cdots$. Hence

$$
m^{\circ}(B) \geqq \lim _{k \rightarrow \infty} \int_{B} f_{k} d \tilde{\mu}=\int_{B} D^{\circ}(, \lambda) d \tilde{\mu} .
$$

THEOREM 11. For each $B \in \Re$,

$$
m^{\circ}(B) \geqq \int_{B} D^{\circ}(, \lambda) d \tilde{\mu} .
$$

Proof. Since $\mu$ is sigma-finite, it suffices to prove the inequality for $\mu(B)$ finite.

The proof from here on is the same, mutatis mutandis, as the proof of Theorem 8 and uses Lemma 7 .

LеммA 8. Let $B \in ß$ and let $\left\{f_{n}\right\}_{n=1}^{\infty}$ be a sequence of non-negative, $\tilde{\mu}$-measurable, real-valued functions defined on $B$ such that

$$
\int_{B} \sup _{n=1}^{\infty} f_{n} d \tilde{\mu}<+\infty \text {. }
$$

Then

$$
\int_{B} \limsup _{n \rightarrow \infty} f_{n} d \tilde{\mu} \geqq \limsup _{n \rightarrow \infty} \int_{B} f_{n} d \tilde{\mu} \text {. }
$$

Proof. Let $g=\sup _{n=1}^{\infty} f_{n}$, let $g_{j}=g-f_{j}$ for $j=1,2, \cdots$, and apply Fatou's Lemma to $\left\{g_{j}\right\}_{j=1}^{\infty}$.

Theorem 12. Suppose $B \in B^{*}$, and suppose $D^{\circ *}(, \lambda)$ is finite almost everywhere on $B(\bmod \tilde{\mu})$. Then, if $\mu$ is regular,

$$
m^{\circ}(B)=\int_{B} D^{\circ}(, \lambda) d \tilde{\mu} .
$$

Proof. It may be supposed that $D^{\circ *}(, \lambda)$ is finite everywhere on $B$. For $k=1,2, \cdots$, let

$$
B_{k}=\left\{t \in B \mid k-1 \leqq D^{\circ *}(t, \lambda)<k\right\} .
$$

Then $\left\{B_{k}\right\}_{k=1}^{\infty}$ is a partitioning of $B$ by members of $B^{*}$. It suffices to show that for $k=1,2, \cdots$,

$$
m^{\circ}\left(B_{k}\right)=\int_{B_{k}} D^{\circ}(, \lambda) d \tilde{\mu} .
$$

Thus we may assume that for some real number $r>0, D^{\circ *}(t, \lambda)<r$ for all $t \in B$. Moreover, by standard arguments we may assume $\tilde{\mu}(B)$ finite (since $\tilde{\mu}$ is sigma-finite). Since $\tilde{\mu}$ is regular, there is an open set $V \subset X$ such that 
$B \subset V$ and $\tilde{\mu}(V)$ is finite. For each $t \in B$ there is an open neighborhood $U_{t} \subset V$ of $t$ in $X$ such that for each $C \in \mathcal{C}$ for which $\varnothing \neq C \subset U_{t}, \lambda(C) / \tilde{\mu}(C)<r$. Let $\mathfrak{u}=\left\{U \mid U=U_{t}\right.$ for some $\left.t \in B\right\}$. Then $u \in \operatorname{cov}(B)$. For $k=1,2, \cdots$, there is some $\mathfrak{u}_{k} \in \operatorname{cov}(B)$ such that $\mathfrak{u}_{k}$ refines $\mathfrak{u}$, such that each member of $\mathfrak{u}_{k}$ has diameter $<1 / k$, and such that

$$
\lambda^{\circ}\left(\mathcal{U}_{k}\right)<m^{\circ}(B)+\frac{1}{k} .
$$

For $k=1,2, \cdots$, there is a sequence $\left\{C_{n}(k)\right\}_{n=1}^{\infty}$ such that $C_{n}(k) \in \mathfrak{e}_{k}$ for each $n$, such that each $C_{n}(k)$ is a subset of a member of $\mathfrak{U}_{k}$, such that $\tilde{\mu}\left(C_{p}(k) \cap C_{n}(k)\right)=0$ if $p \neq n$, and such that

$$
\sum_{n=1}^{\infty} \lambda\left(C_{n}(k)\right)>\lambda^{\circ}\left(\mathcal{U}_{k}\right)-1 / k \geqq m^{\circ}(B)-1 / k .
$$

For $k=1,2, \cdots$, let

$$
f_{k}=\sum_{n=1}^{\infty} \frac{\lambda\left(C_{n}(k)\right)}{\tilde{\mu}\left(C_{n}(k)\right)} C_{C_{n}(k)}
$$

where $C_{C_{n}(k)}$ is the characteristic function of $C_{n}(k)$ on $X$. Then for $k=1,2, \cdots$ and almost every $t \in X(\bmod \tilde{\mu}), f_{k}(t) \leqq r$. By Lemma 8 , since $D^{\circ}(t, \lambda)$ $\geqq \lim \sup _{k \rightarrow \infty} f_{k}(t)$ for almost all $t \in B(\bmod \tilde{\mu})$,

$$
\begin{aligned}
\int_{B} D^{\circ}(, \lambda) d \tilde{\mu} & \geqq \int_{B}\left(\limsup _{k \rightarrow \infty} f_{k}\right) d \tilde{\mu} \\
& \geqq \limsup _{k \rightarrow \infty} \int_{B} f_{k} d \tilde{\mu} \\
& =\limsup _{k \rightarrow \infty} \sum_{n=1}^{\infty} \lambda\left(C_{n}(k)\right) \geqq m^{\circ}(B) .
\end{aligned}
$$

By Theorem 11,

$$
m^{\circ}(B) \geqq \int_{B} D^{\circ}(, \lambda) d \tilde{\mu} .
$$

Thus

$$
m^{\circ}(B)=\int_{B} D^{\circ}(, \lambda) d \tilde{\mu} .
$$

Corollary 12.1. If $D^{\circ *}(, \lambda)$ is finite almost everywhere on $X(\bmod \tilde{\mu})$, then $m^{\circ}(B)=\int_{B} D^{\circ}(, \lambda) d \tilde{\mu}$ for all $B \in \beta^{*}$, and $m^{\circ}$ is continuous with respect to $\tilde{\mu}$. If $D^{\circ *}(, \lambda)$ is finite almost everywhere on $X(\bmod \tilde{\mu})$ and $D^{\circ}(, \lambda)$ is $\tilde{\mu}$ integrable on $X$, then $m^{\circ}$ is absolutely continuous with respect to $\tilde{\mu}$. 
7. Relationship between $m_{0}$ and $m^{\circ}$. Throughout this section, as in $\S 3$, $\mathfrak{e}$ is a $\mu$-Vitali covering of $X$, and $\lambda$ is a non-negative extended-real-valued function whose domain includes $\mathcal{C}$ and which is such that $\lambda(\varnothing)=0$. It will be assumed also that $\mu$ is regular. Thus the theorems of $\$ 5$ and $\S 6$ will apply.

Since $D_{\circ}(, \lambda)$ and $D^{\circ}(, \lambda)$ are non-negative, we may define measures $m_{\circ 0}$ and $m_{0}^{\circ}$ on $\beta^{*}$ by $m_{\circ 0}(B)=\int_{B} D_{\circ}(, \lambda) d \tilde{\mu}, m_{0}^{\circ}(B)=\int_{B} D^{\circ}(, \lambda) d \tilde{\mu}$ for all $B \in B^{*}$. Then $m_{\circ 0}$ (resp., $m_{0}^{\circ}$ ) is continuous with respect to $\tilde{\mu}$ if and only if $D_{\circ}(, \lambda)$ (resp., $D^{\circ}(, \lambda)$ ) is finite almost everywhere $(\bmod \tilde{\mu})$, in which case $m_{\circ 0}$ (resp., $m_{0}^{\circ}$ ) is also sigma-finite by Lemma 5 . If $D_{\circ}(, \lambda)$ is finite almost everywhere $(\bmod \tilde{\mu})$, then (cf. Theorem 9 and Remark 10$), m_{\circ 0}$ is the $\mu$-nonsingular component of $m_{\circ}$.

By Theorems 7 and $11, m_{\circ 0} \leqq m_{0}^{\circ} \leqq m^{\circ}$. In particular, if $m_{\circ}$ is continuous with respect to $\tilde{\mu}$, then, by Theorem $10, m_{\circ}=m_{\circ 0} \leqq m_{0}^{\circ}$. The inequality $m_{\circ} \leqq m^{\circ}$ in case $m_{\circ}$ is continuous with respect to $\tilde{\mu}$ also is a corollary of Theorem 13 below. Finally, $\lambda$ is differentiable with respect to $\mu$ almost everywhere $(\bmod \mu)\left(+\infty\right.$ is allowed as a value of the derivative) if and only if $m_{\circ 0}=m_{0}^{\circ}$.

The relation between $m_{\circ}{ }^{*}$ and $m^{\circ *}$ might be clarified by the study of a more general situation. In the following definition and three lemmas, $\nu_{1} \nu_{1}$, and $\nu_{2}$ are measures on a sigma-ring $\delta$ of subsets of a set $S \in S$, and $\nu_{1}{ }^{*}$ and $\nu_{2}{ }^{*}$ are outer measures on the set of all subsets of $S$ (no relation between $\nu_{j}$ and $\nu_{j}^{*}$ need be assumed). Also, $\tilde{\nu}$ with domain $\tilde{\delta}$ will be the completion of $\nu$.

Definition 11. $\nu_{2}$ (resp., $\nu_{2}^{*}$ ) will be said to $\nu$-dominate $\nu_{1}$ (resp., $\nu_{1}^{*}$ ) if and only if for each $A \in \mathcal{S}$ (resp., $A \subset S$ ) there is some $B \in \mathcal{S}$ such that $\tilde{\nu}(A-B)=0$ and $\nu_{1}(B) \leqq \nu_{2}(A)$ (resp., $\left.\nu_{1}^{*}(B) \leqq \nu_{2}^{*}(A)\right)$.

Lemma 9. Suppose $\nu$ is sigma-finite. Then there is a set $H \in \mathcal{S}$ such that (i) and (ii) below hold.

(i) $\nu_{1}$ (resp., $\nu_{1}^{*}$ or $\left.\left[\nu_{1}^{*} \mid \delta\right]\right)$ is sigma-finite on $H$;

(ii) if $A \in \delta$ is such that $\nu_{1}$ (resp., $\nu_{1}^{*}$ or $\left[\nu_{1}^{*} \mid \delta\right]$ ) is sigma-finite on $A$, then $\nu(A-H)=0$. (Briefly, there is a $\nu$-maximal set $H \in S$ on which $\nu_{1}$ (resp., $\nu_{1}^{*}$ or $\left.\left[\nu_{1}^{*} \mid \delta\right]\right)$ is sigma-finite.)

Proof. Standard arguments reduce the lemma to the case in which $\nu(S)$ is finite. So suppose $\nu(S)$ is finite. (The proof for $\nu_{1}^{*}$ and $\nu_{1}^{*} \mid \delta$ are sufficiently similar to that for $\nu_{1}$ that they will be omitted.) Let

$$
c=\sup \left\{\nu(A) \mid A \in \mathcal{S} \text { and } \nu_{1} \text { is sigma-finite on } A\right\} .
$$

For $n=1,2, \cdots$, there is a set $H_{n} \in S$ such that $\nu_{1}$ is sigma-finite on $A$ and $\nu\left(H_{n}\right)>c-(1 / n)$. Let $H=\bigcup_{n=1}^{\infty} H_{n}$. Then (i) and (ii) may be verified by standard arguments.

Lemma 10. Suppose $\nu$ is sigma-finite, and suppose $\nu_{1}$ or $\nu_{2}$ is sigma-finite (resp., $\nu_{1}^{*} \mid S$ or $\nu_{2}^{*} \mid S$ is sigma-finite and $\nu_{1}^{*} \mid S$ is additive). Suppose $\nu_{2}\left(r e s p ., \nu_{2}^{*}\right)$ $\nu$-dominates $\nu_{1}\left(r e s p ., \nu_{1}^{*}\right)$. Then there is a set $K \in S$ such that $\nu(S-K)=0$ and 
such that $\nu_{1}(Z) \leqq \nu_{2}(Z)$ (resp., $\left.\nu_{1}^{*}(Z) \leqq \nu_{2}^{*}(Z)\right)$ for each $Z \in S$ with $Z \subset K$ (resp., for each $Z \subset K)$.

Proof. The proof for $\nu_{1}$ and $\nu_{2}$ is sufficiently similar to that for $\nu_{1}^{*}$ and $\nu_{2}^{*}$ that the former proof will be omitted. Standard arguments reduce the lemma to the case in which $\nu_{1}^{*}(S)$ or $\nu_{2}^{*}(S)$ is finite, and then $\nu$-domination reduces the lemma to the case in which $\nu_{1}^{*}(S)$ is finite. So suppose $\nu_{1}^{*}(S)$ is finite. Let

$$
c=\sup \left\{\nu_{1}^{*}(A) \mid A \in S \text { and } \nu(A)=0\right\} .
$$

Then $c<+\infty$. For $n=1,2, \cdots$, there is a set $D_{n} \in \mathcal{S}$ such that $\nu\left(D_{n}\right)=0$ and $\nu_{1}^{*}\left(D_{n}\right)>c-(1 / n)$. Let $D=\cup_{n=1}^{\infty} D_{n}$ and $K=S-D$. Then $\nu(S-K)=\nu(D)=0$. Suppose $Z \subset K$. By $\nu$-domination there is $B \in S$ such that $\tilde{\nu}(Z-B)=0$ and $\nu_{1}^{*}(B) \leqq \nu_{2}^{*}(Z)$. There is an $F \in \delta$ such that $Z-B \subset F$ and $\nu(F)=0$. Let $G=(K-B) \cap F$. Then $G \in \mathcal{S}, Z-B \subset G \subset K, G \cap B=\varnothing, G \cap D=\varnothing$, and $\nu(G)=0$. Since $\nu_{1}^{*} \mid S$ is additive, for $n=1,2, \cdots$,

$$
c \geqq \nu_{1}^{*}\left(D_{n} \cup G\right)=\nu_{1}^{*}\left(D_{n}\right)+\nu_{1}^{*}(G) \geqq[c-(1 / n)]+\nu_{1}^{*}(G) .
$$

Hence $\nu_{1}^{*}(G)=0$, and

$$
\nu_{1}^{*}(Z) \leqq \nu_{1}^{*}(B)+\nu_{1}^{*}(Z-B) \leqq \nu_{1}^{*}(B)+\nu_{1}^{*}(G) \leqq \nu_{2}^{*}(Z) .
$$

The lemma is established.

Lemma 11. Suppose $\nu$ is sigma-finite (and $\nu_{1}^{*} \mid S$ is a measure). Suppose $\nu_{2}\left(\right.$ resp., $\left.\nu_{2}^{*}\right) \nu$-dominates $\nu_{1}\left(\right.$ resp., $\left.\nu_{1}^{*}\right)$. Then there is a set $K \in S$ such that for each $A \in \mathcal{S}$ (resp., for each $A \subset S$ ), (i) and (ii) below hold.

(i) $\nu_{1}(A \cap K) \leqq \nu_{2}(A \cap K)$ (resp., $\left.\nu_{1}^{*}(A \cap K) \leqq \nu_{2}^{*}(A \cap K)\right)$;

(ii) neither $\nu_{1}$ nor $\nu_{2}$ (resp., if $A \in \mathbb{B}$, neither $\nu_{1}^{*} \mid S$ nor $\nu_{2}^{*} \mid \delta$ ) is sigma-finite on $A-K$ unless $\tilde{\nu}(A-K)=0$.

Proof. Let $H$ be given by Lemma 9 (resp., Lemma 9 with the parentheses). Apply Lemma 10 with $S$ and $S$ replaced by $H$ and $\{T \mid T \in S, T \subset H\}$ respectively to obtain $K \subset H$. Then (i) and (ii) for each $A \in \mathcal{S}$ (resp., $A \subset S$ ) may be verified.

THEOREM $\left.13{ }^{18}\right) . m^{\circ} \mid \otimes\left(\right.$ resp., $\left.m^{\circ *} \mid B\right) \mu$-dominates $m_{\circ} \mid \otimes\left(\right.$ resp., $\left.m_{\circ}^{*} \mid \otimes\right)$.

Proof. It suffices to prove: if $A \subset X$, then there is a Borel set $B_{0}$ such that $\tilde{\mu}\left(A-B_{0}\right)=0$ and $m_{\circ}\left(B_{0}\right) \leqq m^{\circ *}(A)$.

So suppose $A \subset X$. There are $\mathfrak{u}_{n} \in \operatorname{cov}(A)(n=1,2, \cdots)$ such that $m^{\circ *}(A)=\lim _{n \rightarrow \infty} \lambda^{\circ}\left(\mathcal{U}_{n}\right)$ and such that each member of $\mathfrak{U}_{n}$ has diameter $\leqq 1 / n$. For $n=1,2, \cdots$ there are sets

$$
C_{j}^{n} \in \mathfrak{e}(j=1,2, \cdots)
$$

(18) In this theorem and its proof the assumption that $\mu$ is regular may be dispensed with. A similar statement holds for Theorems 14, 16 (in parts i-iv), 17, 19, Lemma 12, and Corollary 20.1. 
such that $C_{j}^{n} \cap C_{k}^{n}=\varnothing$ for $j \neq k$, such that $C_{j}^{n}$ is a subset of a member of $u_{n}$ for $j=1,2, \cdots$, and such that $\tilde{\mu}\left(A-\bigcup_{j=1}^{\infty} C_{j}^{n}\right)=0$. For $j, n=1,2, \cdots$, there is a set $B_{j}^{n} \in B$ such that $B_{j}^{n} \subset C_{j}^{n}$ and $\tilde{\mu}\left(C_{j}^{n}-B_{j}^{n}\right)=0$. Let

$$
B_{0}=\bigcap_{n=1}^{\infty} \bigcup_{j=1}^{\infty} B_{j}^{n}
$$

Then $\tilde{\mu}\left(A-B_{0}\right)=0$. For $n=1,2, \cdots$,

$$
m_{n}^{*}\left(B_{0}\right) \leqq \sum_{j=1}^{\infty} \lambda\left(C_{j}^{n}\right) \leqq \lambda^{\circ}\left(u_{n}\right)
$$

Hence

$$
m_{\circ}\left(B_{0}\right)=\lim _{n \rightarrow \infty} m_{n}^{*}\left(B_{0}\right) \leqq \lim _{n} \lambda^{\circ}\left(\mathcal{U}_{n}\right)=m^{\circ *}(A)
$$

THEOREM 14. There is a Borel set $K \subset X$ such that for each $A \in \mathbb{B}$ (resp., for each $A \subset X)$ :

(i) $m_{\circ}(A \cap K) \leqq m^{\circ}(A \cap K)\left(\right.$ resp., $\left.m_{\circ}^{*}(A \cap K) \leqq m^{\circ *}(A \cap K)\right)$;

(ii) neither $m_{\circ} \mid B$ nor $m^{\circ} \mid \mathbb{B}$ (if $A \in B$ ) is sigma-finite on $A-K$ unless $\tilde{\mu}(A-K)=0$.

Proof. Apply Theorem 14 and Lemma 11.

In the following theorem the possibility $D_{\circ}(t, \lambda)=D^{\circ}(t, \lambda)=D(t, \lambda)=+\infty$ for all $t$ in some set of positive $\tilde{\mu}$-measure is not excluded. A measure $\nu$ on $B$ is $\mu$-Lipschitzian if and only if $\nu \leqq c \mu$ for some real $c$ (cf. Definition 4).

THEOREM 15. Suppose $m_{\circ} \mid B \geqq \nu$ for every $\mu$-Lipschitzian measure $\nu$ on $B$ such that $m^{\circ} \mid \otimes \geqq \nu$ (e.g., suppose $\left.m_{\circ}\left|B \geqq m^{\circ}\right| \otimes\right)$. Then $\lambda$ is differentiable almost everywhere $(\bmod \mu)$.

Proof. Suppose the theorem is false. There is a set $B_{0} \in \circledast$ such that $\mu\left(B_{0}\right)$ $>0$ and $D_{\circ}(t, \lambda)<D^{\circ}(t, \lambda)$ for all $t \in B_{0}$. There are a set $A \in B$ and real numbers $r>0$ and $\delta>0$ such that $A \subset B_{0}, 0<\mu(A)<+\infty$, and $D_{0}(t, \lambda) \leqq r$ and $D^{\circ}(t, \lambda)>D_{\circ}(t, \lambda)+\delta$ for all $t \in A$. Define

$$
\nu(B)=\int_{A \cap_{B}}\left[D_{\circ}(, \lambda)+\delta\right] d \tilde{\mu}
$$

for each $B \in B$. Then $\nu$ is a measure on $B, \nu \leqq(r+\delta) \mu$, and

$$
\nu(B) \leqq \int_{A \cap_{B}} D^{\circ}(, \lambda) d \tilde{\mu} \leqq m^{\circ}(A \cap B) \leqq m^{\circ}(B)
$$

for each $B \in B$ by Theorem 11. Since $\nu \leqq m^{\circ}\left|\otimes, \nu \leqq m_{\circ}\right| \otimes$. Hence, by Theorem 6 , 


$$
\begin{aligned}
\nu(A) & \leqq \int_{A} D_{\circ}(, \lambda) d \tilde{\mu}<\int_{A} D_{\circ}(, \lambda) d \tilde{\mu}+\delta \tilde{\mu}(A) \\
& =\int_{A}\left[D_{\circ}(, \lambda)+\delta\right] d \tilde{\mu}=\nu(A)
\end{aligned}
$$

(for $\left.\int_{A} D_{\circ}(, \lambda) d \tilde{\mu} \leqq r \mu(A)<+\infty\right)$, a contradiction. Thus the theorem is established.

Corollary 15.1. Suppose $D^{\circ} *(t, \lambda)<+\infty$ for almost every $t \in X(\bmod \mu)$. Then $\lambda$ is differentiable almost everywhere $(\bmod \tilde{\mu})$ with respect to $\mu$ and $\mathfrak{e}$ if and only if $m_{\circ}\left|B \geqq m^{\circ}\right| Q$.

Proof. If $\lambda$ is differentiable almost everywhere $(\bmod \tilde{\mu})$, then $m_{\circ}\left|\cap \geqq m^{\circ}\right| \cap$ by Theorem 9 and Corollary 12.1. For the converse, apply Theorem 15.

Lemma 12. Suppose $\nu$ is a measure on a sigma-ring $S$ with $B \cup \mathcal{C} \subset \mathcal{S} \subset B^{*}$ such that $\nu$ is continuous with respect to $\tilde{\mu} \mid \mathcal{S}$ and such that $\nu \mid \mathcal{C} \leqq \lambda$. Then $\nu \leqq m^{\circ *} \mid$ S.

Proof. Suppose $B \in \mathcal{S}$ and $\mathcal{u} \in \operatorname{cov}(B)$. There are sets $C_{n} \in \mathcal{C}(n=1,2, \cdots)$ such that each $C_{n}$ is a subset of a member of $u$, such that $C_{j} \cap C_{h}=\varnothing$ if $j \neq h$, and such that

$$
\tilde{\mu}\left(B-\bigcup_{n=1}^{\infty} C_{n}\right)=0
$$

Then

$$
\begin{aligned}
\nu(B) & \leqq \nu\left(\bigcup_{n=1}^{\infty} C_{n}\right)=\sum_{n=1}^{\infty} \nu\left(C_{n}\right) \\
& \leqq \sum_{n=1}^{\infty} \lambda\left(C_{n}\right) \leqq \lambda^{\circ}(\mathcal{u}) .
\end{aligned}
$$

Hence, for each $B \in \mathcal{S}$,

$$
\nu(B) \leqq \inf _{\mathcal{U} \in \operatorname{cov}(B)} \lambda^{\circ}(\mathcal{U})=m^{\circ *}(B) .
$$

The theory of upper and lower measures induced by a measure is done in the following theorem; (v)-(vii) are variants of known results (cf., e.g., Hahn-Rosenthal [2] and Morse [5; 6]). Differentiability is taken with respect to $\mathcal{C}$ and $\mu$.

THEOREM 16. Suppose $\lambda$ is a measure on a sigma-ring $\mathcal{S}$ with $B \cup \mathcal{C} \subset \mathcal{S} \subset B^{*}$. Then (i)-(vii) below hold.

(i) $\lambda \leqq m_{0} * \mid s$.

(ii) If $\lambda$ is continuous with respect to $\tilde{\mu} \mid \mathrm{s}$, then $\lambda \leqq m^{\circ *} \mid \mathrm{s}$. 
(iii) If $\lambda$ is regular, then $m^{\circ} * \mid \S \leqq \lambda$, and each measure $\nu$ on $\delta$ which is $\leqq \lambda$ and continuous with respect to $\tilde{\mu} \mid S$ is $\leqq m^{\circ} * \mid S$.

(iv) If $\lambda$ is regular and continuous with respect to $\tilde{\mu} \mid \delta$, then $m^{\circ *} \mid \delta=\lambda$.

(v) $\lambda$ is differentiable at almost every $t \in X(\bmod \mu)$ for which $\lambda(N-\{t\})$ is finite for some neighborhood $N$ of $t$ in $X$.

(vi) If $\lambda$ is regular, $\lambda$ is differentiable almost everywhere $(\bmod \mu)$.

(vii) If, where $C^{\circ}$ is the interior of $C, \tilde{\mu}\left(C^{\circ}\right)=\tilde{\mu}(C)$ for each $C \in \mathfrak{e}$, then $\lambda$ is differentiable almost everywhere $(\bmod \mu)$.

Proof. (i) follows trivially from the definition of $m_{0} *$. (ii) follows from Lemma 12 with $\nu=\lambda$.

To prove (iii), suppose $\lambda$ is regular. Suppose $S \in S$, and suppose $\epsilon>0$. There is an open set $V \subset X$ such that $S \subset V$ and $\lambda(V)<\lambda(S)+\epsilon$. Then $\{V\} \in \operatorname{cov}(S)$, and it is easily verified that $\lambda^{\circ}(\{V\}) \leqq \lambda(V)$. Hence

$$
m^{\circ *}(S)=\inf _{\mathcal{U} \in \operatorname{cov}(S)} \lambda^{\circ}(\mathcal{U}) \leqq \lambda^{\circ}(\{V\}) \leqq \lambda(V) \leqq \lambda(S)+\epsilon .
$$

Hence $m^{\circ *}(S) \leqq \lambda(S)$ for each $S \in \mathcal{S}$. Part of (iii) is established. The other part follows from Lemma 12.

(iv) follows from (ii) and (iii).

To prove (v), let $X_{[1]}$ be the set of all $t \in X$ for which $\lambda(N-\{t\})$ is finite for some neighborhood $N$ of $t$ in $X$. Then $X_{[1]}$ is open. Let $\lambda_{[1]}=\lambda \mid S_{[1]}$ where $s_{[1]}=\left\{S \mid S \in S, S \subset X_{[1]}\right\}$, let $\mu_{[1]}=\mu \mid \otimes_{[1]}$ where $B_{[1]}$ is the set of all Borel sets of $X_{[1]}$, and let $\mathfrak{e}_{[1]}=\left\{C \mid C \in \mathfrak{e}, C \subset X_{[1]}\right\}$. Then $\mathfrak{e}_{[1]}$ is a $\mu_{[1]}$-Vitali covering of $X_{[1]}$ with $\lambda_{[1]}$ and $\mu_{[1]}$ regular measures on $X_{[1]}$ (cf. Remark 9, §5). Let $m_{\text {o[1] }}$ and $m_{[1]}^{\circ}$ be the lower and upper measures induced by $\lambda_{[1]}$ and $\mathfrak{e}_{[1]}$. Then $m_{[1]}^{\circ} \leqq \lambda_{[1]} \leqq m_{\text {o[1] }}$ on $B_{[1]}$ by (iii) and (i). Hence $\lambda_{[1]}$ is differentiable with respect to $\mathfrak{e}_{[1]}$ and $\mu_{[1]}$ almost everywhere $\left(\bmod \mu_{[1]}\right)$ in $X_{[1]}$ by Theorem 15 . Hence $\lambda$ is differentiable with respect to $\mathfrak{C}$ and $\mu$ almost everywhere $(\bmod \mu)$ in $X_{[1]}$. Thus (v) is established. (vi) follows from ( $v$ ).

To prove (vii) let

$$
\begin{aligned}
& D_{n}=\left\{C \mid C \in \mathbb{C}_{n}, \lambda(C)<+\infty\right\} \quad(n=1,2, \cdots), \\
& D_{0}=\bigcap_{n=1}^{\infty} \bigcup_{C \in D_{n}} C .
\end{aligned}
$$

There is a sequence $\left\{C_{j}\right\}_{j=1}^{\infty}$ of members of $\cup_{n=1}^{\infty} D_{n}$ such that $C_{j} \cap C_{h}=\varnothing$ for $i \neq h$ and

$$
\tilde{\mu}\left(D_{0}-\bigcup_{n=1}^{\infty} C_{n}\right)=0 .
$$

Suppose $\mu\left(C^{\circ}\right)=\tilde{\mu}(C)$ for each $C \in \mathcal{C}$. Since $\mu\left(C_{n}^{\circ}\right)<+\infty$ for $n=1,2, \cdots, \lambda$ is differentiable almost everywhere in $\bigcup_{n=1}^{\infty} C_{n}^{\circ}$ by $(v)$, hence almost every- 
where in $\cup_{n=1}^{\infty} C_{n}$, and hence almost everywhere in $D_{0}$. Trivially, $D(t, \lambda)=+\infty$ for each $t \in X-D_{0}$. Thus (vii) is established.

The theorem is proved.

Many classical examples of $\mu$-Vitali coverings have the following property $P$.

Definition 12. We shall say that $\mathfrak{e}$ has property $P$ if and only if for all $A \subset X$ and $u \in \operatorname{cov}(A)$, there is a sequence $\left\{C_{j}\right\}_{j=1}^{\infty}$ of members of $\mathcal{e}$ such that $\tilde{\mu}\left(C_{j} \cap C_{k}\right)=\varnothing$ for $j \neq k$, such that each $C_{j}$ is a subset of a member of $\mathcal{u}$, and such that $A \subset \cup_{j=1}^{\infty} C_{j}$.

Theorem 17. Suppose e has property $P$. Then $m_{\circ}{ }^{*} \leqq m^{\circ *}$.

Proof. Given $A, \mathcal{u}$, and $\left\{C_{j}\right\}_{j=1}^{\infty}$ as in Definition 12, we have

$$
\lambda_{\circ}(\mathcal{u} ; A) \leqq \sum_{j=1}^{\infty} \lambda\left(C_{j}\right) \leqq \lambda^{\circ}(\mathcal{u}) .
$$

Hence

$$
\begin{aligned}
m_{\circ}^{*}(A)=\bar{m}_{\circ}^{*}(A) & =\lim _{\mathcal{U} \in \operatorname{cov}(A)} \lambda_{\circ}(\mathcal{U} ; A) \\
& \leqq \lim _{\mathcal{U} \in \operatorname{cov}(A)} \lambda^{\circ}(\mathcal{U}) \\
& =m^{\circ *}(A) .
\end{aligned}
$$

Definition 13. If $\lambda$ is such that $m_{\circ}\left|B=m^{\circ}\right| B$, we say that $m=m_{\circ} \mid B$ $=m^{\circ} \mid B$ is the $\lambda$-determined measure on $B$.

THEOREM 18. Suppose $\mathfrak{e}$ has property $P$ and $D^{\circ *}(t, \lambda)<+\infty$ for almost every $t \in X(\bmod \mu)$. Then $\lambda$ is differentiable almost everywhere $(\bmod \tilde{\mu})$ with respect to $\mu$ and $\mathfrak{C}$ if and only if $m_{\circ}\left|B=m^{\circ}\right| B$, i.e., if and only if there is a $\lambda$-determined measure on $B$.

Proof. Apply Theorem 17 and Corollary 15.1.

TheOREM 19. Suppose e has property $P$, and suppose $\lambda$ is a regular measure on a sigma-ring $\delta$ with $B \cup \mathcal{C} \subset S \subset B^{*}$. Then $m_{\circ}^{*}\left|\delta=m^{\circ}\right| \delta=\lambda$, and $\lambda \mid \otimes$ is the $\lambda$-determined measure on $B$.

Proof. Apply Theorem 17 and (iii) and (i) of Theorem 16 to obtain $m_{\circ}^{*}\left|\delta \leqq m^{\circ *}\right| \delta \leqq \lambda \leqq m_{\circ}^{*} \mid s$.

The following theorem follows from the definitions of $m^{\circ *}$ and $m_{0}^{*}=\bar{m}_{\circ} *$ and is of interest mainly if $\mathfrak{C}$ has property $P$.

Theorem 20. Suppose there is a $\lambda$-determined measure $m$ on $B$. Then for each $B \in Q$ with $m(B)$ finite and each real $\epsilon>0$ there is some $u \in \operatorname{cov}(B)$ such that: if $C_{j} \in \mathcal{C}$ is a subset of a member of $u$ for $j=1,2, \ldots, \tilde{\mu}\left(C_{j} \cap C_{k}\right)=0$ for $j \neq k$, and $B \subset \cup_{j=1}^{\infty} C_{j}$, then 


$$
\left|m(B)-\sum_{j=1}^{\infty} \lambda\left(C_{j}\right)\right|<\epsilon .
$$

Corollary 20.1. Suppose there is a $\lambda$-determined measure $m$ on $\circledast$. Then for each compact $B \in B$ with $m(B)$ finite and each real $\epsilon>0$ there is some real $\delta>0$ such that: if $C_{j} \in \mathcal{C}$ meets $B$ and has diameter $<\delta$ for $j=1,2, \cdots$, $\tilde{\mu}\left(C_{j} \cap C_{k}\right)=0$ for $j \neq k$, and $B \subset \cup_{j=1}^{\infty} C_{j}$, then

$$
\left|m(B)-\sum_{j=1}^{\infty} \lambda\left(C_{j}\right)\right|<\epsilon .
$$

Proof. Let $u$ be given by Theorem 20 . By standard methods $\left({ }^{19}\right)$ there is a real $\delta>0$ such that each subset of $X$ meeting $B$ and having diameter $<\delta$ is a subset of a member of $u$. The conclusion of Corollary 20.1 follows.

8. Specialization to Lebesgue measure in $R^{q}$. Lebesgue measure in $R^{q}$ will be written $L_{q}$. All intervals in $R^{1}$ will be considered to be bounded and to contain more than one point. A closed interval in $R^{q}$ is a cartesian product $\prod_{j=1}^{q} J_{j}$ of closed intervals $J_{j}$ in $R$; if $J_{j}$ has length $a$ for $j=1, \cdots, q$, then $\prod_{j=1}^{q} J_{j}$ is a closed cube of edge-length $a$. A closed $j$-simplex in $R^{q}$ is the convex hull of any set of $j+1$ affinely independent points of $R^{q}$, the vertices of the simplex.

Definition 14. Suppose $A \subset R^{q}$ is bounded, Lebesgue measurable, and contains more than one point. The regularity of $A$ is defined to be

$$
r(A)=L_{q}(A) /[\operatorname{diam}(A)]^{q} .
$$

Suppose $S$ is a closed $q$-simplex in $R^{q}$, and suppose $J \subset R$ is a closed interval. It is well-known that there is a "decomposition" $D$ of $S \times J \subset R^{a+1}$ such that $D$ is a set of closed $(q+1)$-simplices for which (1)-(4) below hold $\left({ }^{20}\right)$.

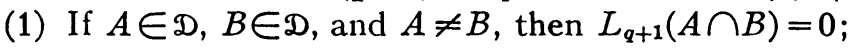

(2) $S \times J=\cup_{A \in \mathfrak{D}} A$;

(3) $D$ has exactly $q+1$ members;

(4) if $A \in D$, then

$$
L_{q+1}(A)=\frac{1}{q+1} L_{1}(J) \cdot L_{q}(S)=\frac{1}{q+1} L_{q+1}(S \times J) .
$$

This may be used to prove the following well-known lemma by induction on the dimension.

Lemma 13. Suppose $H$ is a closed interval in $R^{q}$. Then there is a set $\mathfrak{N}$ of $q$-simplices such that (1)-(4) below hold.

(1) If $A \in \mathfrak{N}, B \in \mathfrak{M}$, and $A \neq B$, then $L_{q}(A \cap B)=0$.

(19) Cf. Eilenberg and Steenrod [1, p. 65] for the method of proof.

${ }^{(20)}$ Cf. Eilenberg and Steenrod $[1$, p. 70$]$ for a description. 
(2) $H=\bigcup_{A \in \mathfrak{T C}} A$.

(3) IT has exactly $q$ ! members.

(4) If $A \in \mathfrak{T}$, then $L_{q}(A)=L_{q}(H) / q$ ! and $r(A) \geqq r(H) / q$ !

If $H$ is a cube in $R^{a}$ of edge-length $a$, then trivially

$$
r(H)=a^{q} /\left(\left(q a^{2}\right)^{1 / 2}\right)^{q}=q^{-q / 2} .
$$

Definition 15. A sigma-interval in $R^{q}$ is any subset of $R^{q}$ which is the union of countably many closed intervals.

Any closed interval in $R^{q}$ is a sigma-interval. Any open subset of $R^{q}$ is a sigma-interval ${ }^{(21)}$.

Theorem 21. Suppose $X$ is a sigma-interval in $R^{a}, B$ is the set of all Borel sets of $X$, and $\mu=L_{q} \mid$ B. Suppose $c$ is a real number such that $0<c<q^{-q / 2}$. Suppose (a) or (b) or (c) below holds.

(a) $\mathfrak{C}$ is the set consisting of $\varnothing$ and all closed intervals of $X$ of regularity $>c$.

(b) $\mathcal{C}$ is the set consisting of $\varnothing$ and all closed $q$-simplices of $X$ of regularity $>c / q$ !

(c) $\mathcal{C}$ is the set consisting of $\varnothing$ and all compact subsets of $X$ of regularity $>c$.

Then $\mathfrak{C}$ is a $\mu$-Vitali covering of $X$ having property $P$. Hence, if $\lambda$ is a nonnegative extended-real-valued function whose domain includes $\mathfrak{e}$ such that $\lambda(\varnothing)=0$, then all the theorems of $\S \S 2-7$ are valid (for the conditions placed on $X$, $\Theta, \mu$, and $\mathcal{C}$ within $\S \S 2-7$ are valid).

Proof. We first prove (1)-(3) of Definition 1. (1) and (2) are trivial.

To prove (3) we write $X=\cup_{k=1}^{\infty} J_{k}$, each $J_{k}$ being a closed interval. Consider a positive integer $n$. Each $J_{k}$ is the union of finitely many cubes of diameter $<1 / n$ and regularity $>c$, and each of these cubes is (by Lemma 13) the union of finitely many simplices of regularity $>c$. Thus (3) of Definition 1 holds.

By Remark 2 of $\S 2, \mathcal{e}$ is a $\mu$-Vitali covering of $X$.

It remains to prove that $\mathcal{C}$ has property $P$.

Suppose $A \subset X$ and $u \in \operatorname{cov}(A)$. There is a sequence $\left\{J_{n}\right\}_{n=1}^{\infty}$ of closed intervals in $R^{a}$ such that $X=\cup_{n=1}^{\infty} J_{n}$. For $n=1,2, \cdots$, we will arrive at a set $\mathscr{J}(n)$ of closed intervals in $R^{q}$ such that (a)-(d) below hold where $U_{0}$ $=\bigcup_{U \in \mathcal{U}} U$.

(a) $U_{J \in \mathcal{J}(n)} J=J_{n} \cap\left(U_{0}-U_{j<n} J_{j}\right)$.

(b) If $J \in \mathcal{J}(n), J^{\prime} \in \mathcal{J}(n)$, and $J \neq J^{\prime}$, then $\mu\left(J \cap J^{\prime}\right)=0$.

(c) Each member of $\mathcal{J}(n)$ is a subset of a member of $\mathcal{u}$.

(d) If $J \in \mathcal{J}(n), r(J)>c$.

Since $U_{0}-U_{j<n} J_{j}$ is open in $X$, by standard methods $\left({ }^{22}\right)$ there are sets $g^{\prime}(n)$ of closed intervals such that (a) and (b) hold for $g(n)=g^{\prime}(n)$. For each

(21) Cf. Munroe $[7$, p. 126] for the method of proof (there applied to half-open intervals).

(22) Cf. Munroe $[7$, p. 126] for the method of proof. 
$J \in \mathcal{J}^{\prime}(n)$ there is a number $\delta(J)>0$ such that each subset of $J$ of diameter $<\delta(J)$ is a subset of a member of $\mathcal{u}\left({ }^{23}\right)$. By elementary means one may establish the existence for each $J \in \mathcal{J}^{\prime}(n)$ of a finite set $\mathcal{J}(J)$ of closed intervals of diameter $\left\langle\delta(J)\right.$ and regularity $>c$ such that $J=\bigcup_{H \in \mathcal{I}(J)} H$ and such that $\mu\left(H \cap H^{\prime}\right)=0$ if $H, H^{\prime} \in g(J)$ and $H \neq H^{\prime}$. Let

$$
\mathcal{J}(n)=\bigcup_{J \in \mathcal{J}^{\prime}(n)} \mathcal{J}(J) .
$$

Then (a)-(d) may be verified. Let $\mathfrak{F}=\bigcup_{n=1}^{\infty} \mathcal{J}(n)$. By Lemma 13 for each $H \in \mathfrak{F C}$ there is a finite set $\mathfrak{M}(H)$ of closed simplices satisfying (1)-(4) of Lemma 13 with $\mathfrak{N}=\mathfrak{T}(H)$. Let $\Re=\bigcup_{H \in \mathcal{K}} \mathfrak{T}(H)$. Then $\mathcal{K}$ and $\Re$ are countable and refine $u, A \subset \bigcup_{H \in \mathcal{K}} H=\bigcup_{K \in \mathscr{K}} K$, the intersection of any two distinct members of $\mathfrak{H C}$ has $\mu$-measure 0 , the intersection of any two distinct members of $\mathfrak{K}$ has $\mu$-measure 0 , and either $\mathfrak{K C} \mathcal{C}$ or $\mathfrak{K} \subset \mathbb{C}$.

Hence $\mathcal{C}$ has property $P$.

\section{REFERENCES}

1. S. Eilenberg and N. Steenrod, Foundations of algebraic topology, Princeton, Princeton University Press, 1952.

2. H. Hahn and A. Rosenthal, Set functions, Albuquerque, University of New Mexico Press, 1948.

3. P. R. Halmos, Measure theory, New York, D. Van Nostrand, 1950.

4. W. K. Moore, The characterizations of a class of transformations and a class of differentiable functions, University of Kansas, 1951 (unpublished doctoral dissertation).

5. A. P. Morse, $A$ theory of covering and differentiation, Trans. Amer. Math. Soc. vol. 55 (1944) pp. 205-235.

6. - Perfect blankets, Trans. Amer. Math. Soc. vol. 61 (1947) pp. 418-442.

7. M. E. Munroe, Introduction to measure and integration, Reading, Mass., AddisonWesley, 1953.

8. T. Radó, Length and area, Amer. Math. Soc. Colloquium Publications, vol. 30, New York, 1948.

College of the Holy Cross, WORCESTER, MASSACHUSETTS

UNIVERSITY OF KANSAS, LAWRENCE, KANSAS

(23) Cf. Eilenberg and Steenrod [1, Lemma 7.5, p. 65]. 